\title{
Lethal and atypical fetopathy caused by cytomegalovirus recurrence with isolated intra-abdominal complication in
} an immune woman

\author{
Pichon $\mathrm{M}^{1,2^{*}}$, Gaymard $\mathrm{A}^{1,2}$, Bolze $\mathrm{PA}^{3}$, Gaucherand $\mathrm{P}^{3}$ and Mekki $\mathrm{Y}^{1}$ \\ ${ }^{1}$ Hospices Civils de Lyon, Laboratoire de Virologie, Institut des Agents infectieux, CBN, Groupement Hospitalier Nord, Lyon, France \\ ${ }^{2}$ Univ Lyon, VirPath, CIRI, INSERM U1111, CNRS UMR5308, ENS Lyon, Université Claude Bernard Lyon 1, Lyon, France \\ ${ }^{3}$ Hospices Civils de Lyon, Service de Gynécologie et Oncologie, Groupement Hospitalier Sud, Lyon, France
}

\begin{abstract}
A pregnant woman did not show any problem during the first trimester, but ultrasound exam, confirmed by RMI, showed a large intra-abdominal hyper-echogenic cystic image at 24 weeks of gestation. Meconial peritonitis was suspected, complicated by stable splenomegaly. No neurological signs, variation of the amniotic fluid volume, cardiologic dysfunction or thrombocytemia was observed. Serological assays done before and during pregnancy showed constant low IgM and high IgG antiCMV levels. Amniocentesis showed a high viral load in amniotic fluid. Fetal and maternal bleed were positive. The pregnancy was interrupted at 31 weeks without post-mortem fetal examination. Placenta revealed hydrophic villi with cytomegalic anti-CMV positive inclusions. This case of CMV recurrence leads to severe complications of the fetal development in a pregnant woman. The isolated abdominal abnormalities were an atypical presentation of fetal CMV, causing inappropriate decisions. Multiplex examinations on amniotic fluid would be keys during the pregnancy examination to prevent fatal outcomes.
\end{abstract}

\section{Case report}

A 23-year-old first gravida, non-para woman was referred to the Department of Obstetrics and Prenatal diagnosis for diagnosis of a fetal intra-abdominal abnormality found during ultrasound examination. Her medical history showed no abnormalities after a spontaneous pregnancy and she declared no symptoms at the beginning of her following. During her first trimester, no clinical or biological signs were identified, the bio-markers (alpha-feto protein, beta-HCG) associated to a normal ultrasound concluded to a risk less than $1 / 10,000$ of Down syndrome.

The pregnancy was monitored until the $24^{\text {th }}$ week of amenorrhea (WoA), when ultrasound examination showed the presence of important intra-abdominal image. This image, unique and cystic, estimated to $6.4 \mathrm{~cm}$ X $3.4 \mathrm{~cm}$ (Figure 1A) contained radial internal partitions, was echogenic and was associated to splenomegaly. Neither hydramnios nor peritoneal effusion was found during examination. Placenta was normal without any detachment. On the supradiaphragmatic region, a cardiomegaly was observed without functional stir on ventricular function (Figure 1C).

A systematic first-line panel, orientated by ultrasound symptomatology, was prescribed by obstetricians and did not demonstrated abnormalities. Cystic fibrosis most frequent mutations searched in the two parents' blood were not found. Fetal karyotype showed a female genotype without chromosomal structure or number abnormalities. Viral main serologies were performed on maternal blood and showed immunoglobulin G (IgG) for Rubella and cytomegalovirus (CMV) and no immunization for HIV, toxoplasma, hepatitis viruses and Syphilis (Liaison ${ }^{\circ}$, Diasorin, Italy) (Table 1A). No active infections, represented by $\operatorname{IgM}$, were found at this moment of the clinical course.
Because of the maternal blood group (A negative), Kleihauer test was made looking for materno-fetal hemorrhage but remained negative.

Clinicians decided to strictly monitor this cystic image of undetermined nature with consultation and ultrasound surveillance. A materno-fetal MRI was also realized to identify precisely this abnormality (Figure 1D and 1E). This MRI concluded to a meconium cyst associated to a digestive atresia and uro-genital system repression. Amniotic fluid was present in normal quantity (no oligamnios or hydramnios). No abnormalities of the neurological system were reported.

After these investigations, it was decided to strictly monitor the radiological images by ultrasound and MRI. An intermediate consultation for diminution of fetus movement allows the diagnosis of Gardnerella infection without consequence on the pregnancy but lead to an intensification of the monitoring. At $30 \mathrm{WoA}$, clinicians asked for viral research on amniotic fluid, punted because of the non-resorption of the cystic image modified (Figure 1B). On the amniotic fluid, the virology laboratory found a very high load of CMV (more than 32 million copies/ml) (Argene, bioMérieux, France). This virus was also tested positive in fetal and maternal blood (Argene, bioMérieux, France). Absence of Parvovirus B19 was confirmed in amniotic fluid and in fetal blood (Argene, bioMérieux, France) (Table 1B). No other signs that an increase in neutrophils population $\left(\mathrm{XN} 9000^{\circ}\right.$, Sysmex,

*Correspondence to: Maxime Pichon, Department of Virology, Infectious Agents Institute, 103 grande rue de la Croix Rousse, 69003 Lyon, France, Tel: +33(0)472071022 ; E-mail: maxime.pichon01@chu-lyon.fr

Key words: $C M V$, recurrence, fetopathy, echography, hyperechogenic bowel

Received: April 02, 2018; Accepted: April 24, 2018; Published: April 27, 2018 

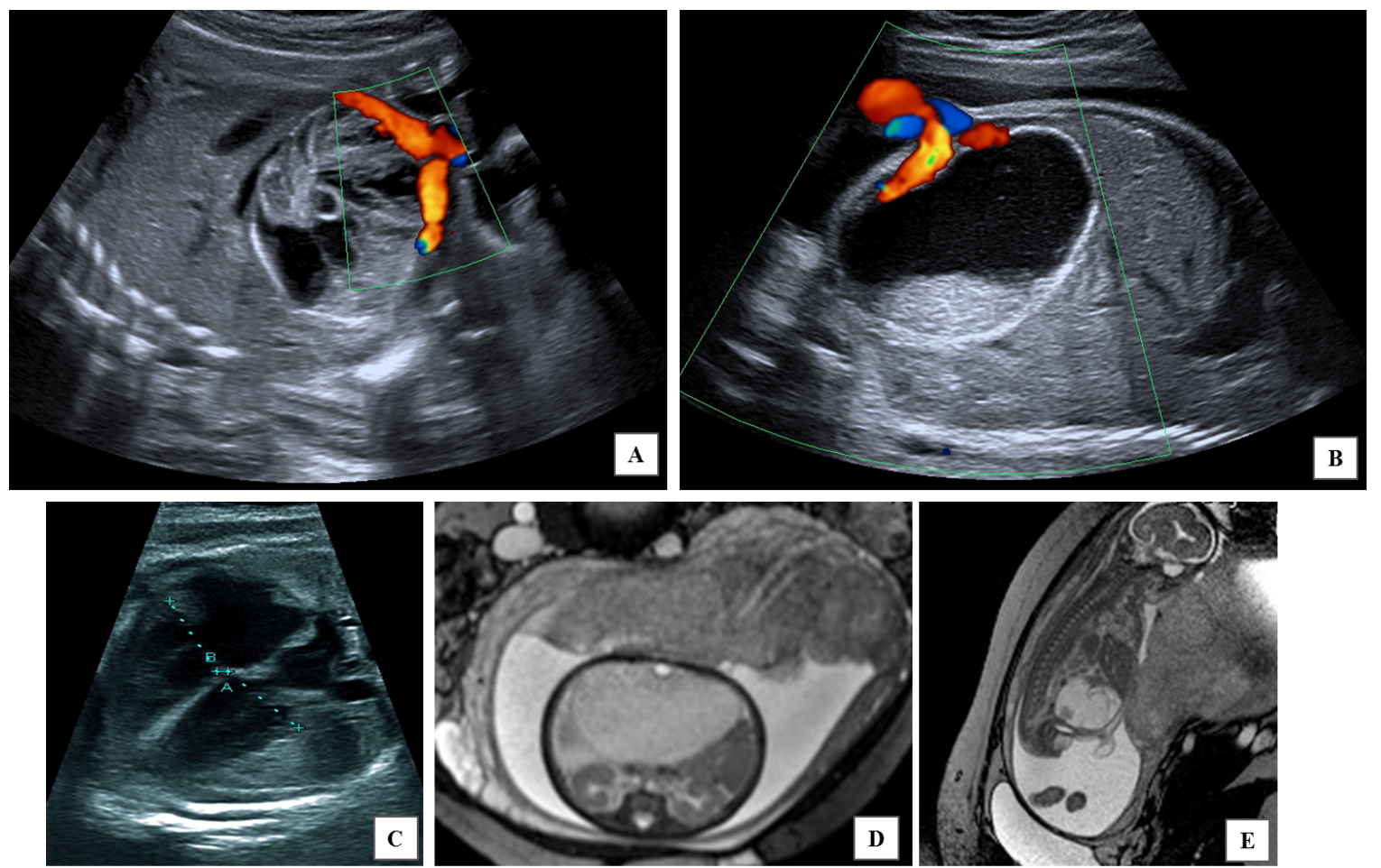

Figure 1. Radiological examination of the pregnancy. A) Longitudinal section of ultrasound examination with Doppler after 24 weeks of pregnancy. Ultrasound examination observed a heterogeneous cyst with echogenic septum. Doppler examination confirmed that no vascularization has been associated to this image. B) Transversal section of ultrasound examination with Doppler after 29 weeks of pregnancy. Ultrasound examination confirmed the presence of a cystic image. This image remained stable, heterogeneous and non-vascularized since its first observation. This term allowed sonographer to discover echogenicity levels modified by the position of the pregnant woman. C) Ultrasound examination of fetal heart after 29 weeks of pregnancy. D) Sagittal section at the fetal abdominal level in T1 MRI sequence. E) Transversal section of the fetus in TI MRI sequence. A hyper signal T1 cyst with heterogeneous content could be observed in fetal abdomen. No impact was observed on fetal heart and kidney. No abnormal quantity of amniotic level has been put in evidence.

Table 1. Biological surveillance of the pregnancy. A) Quantification of anti-CMV antibodies viral load before and during the pregnancy. * Serology performed using UniCel ${ }^{\circ}$ DxI (Beckman Coulter, Switzerland). ** Serology performed using Liaison ${ }^{\circledR}$ (Diasorin, Italy). ${ }^{\circledR}$ Serology performed before pregnancy beginning. B) Quantification of CMV and PvB19 viral load during and after the pregnancy. Quantification has been performed using CMV kit (Vela diagnostic,Singapore) for viremias and R-gene CMV kit (bioMérieux, France) for viral load in amniotic fluid and placenta biopsies. ๆ correspond to quantification at the day of medical interruption of the pregnancy. NP: non-performed analyses.

A

\begin{tabular}{|c|c|c|c|c|c|c|}
\hline Sampling dat & \multicolumn{2}{|c|}{ 08/19/15*® } & $09 / 22 / 15^{*}$ & \multicolumn{2}{|c|}{$03 / 23 / 16 * *$} & $04 / 27 / 16 * *$ \\
\hline \multicolumn{2}{|l|}{ IgG } & $381 \mathrm{aU} / \mathrm{ml}$ & $402 \mathrm{aU} / \mathrm{ml}$ & \multicolumn{2}{|c|}{$245,6 \mathrm{aU} / \mathrm{ml}$} & $229,1 \mathrm{aU} / \mathrm{ml}$ \\
\hline \multicolumn{2}{|l|}{ IgM } & $0 \mathrm{aU} / \mathrm{ml}$ & $0 \mathrm{aU} / \mathrm{ml}$ & \multicolumn{2}{|c|}{$0 \mathrm{aU} / \mathrm{ml}$} & $0 \mathrm{aU} / \mathrm{ml}$ \\
\hline \multicolumn{7}{|l|}{ B } \\
\hline & \multicolumn{5}{|c|}{ CMV viral load (copies/ml) } & $\begin{array}{l}\text { PvB19 Viral Load } \\
\text { (copies/ml) }\end{array}$ \\
\hline Sampling date & 03/11/16 & 03/16/16 & 03/30/16 & $04 / 06 / 16^{4}$ & $04 / 08 / 16$ & $04 / 06 / 16$ \\
\hline Maternal Blood & NP & NP & 0 & 198 & 0 & NP \\
\hline Amniotic fluid & 37870058 & 19107073 & NP & 32026698 & NP & 0 \\
\hline Fetal Blood & NP & NP & NP & 1200000 & NP & 0 \\
\hline Placenta & NP & NP & NP & 658000 & NP & NP \\
\hline
\end{tabular}

Japan) in maternal blood was in favor of an inflammation. This diagnosis of maternofoetal CMV infection, associated to a surge of the cystic image with mechanical stir on supra diaphragmatic organs (cardiomegaly), led to a termination of the pregnancy proposition, on parents' demand. The termination led to the expulsion of a stillborn at 31 weeks of amenorrhea.

Retrospective examination of the sera used to declare pregnancy demonstrated that woman was immune to CMV previously to the beginning of her pregnancy (IgG-positive and IgM-negative) (UniCel ${ }^{\circ}$ DxI, Beckman Coulter, Switzerland), so the CMV-infection is due to a maternal recurrence. Despite of this statement, it was decided to examine all biological maternal and fetal samples to determine the moment of the transmission by determination of immunoglobulin quantification and CMV viral load (Table 1A). Examination of the placenta by pathologists confirmed the presence of cytopathic effects consistent with a CMV infection (Figure 2) showing typical macroand microscopic aspect and positive anti-CMV staining. Moreover, viral load on placenta was estimated to a tenth of the viral load in amniotic fluid (Table 1B). Finally, Beta-2-microglobulin, considered as a possible biomarker for pejorative issues, was quantified on amniotic fluid puncted at $30 \mathrm{WoA}$ and was not elevated compared to values issued from the cohort of Cagdas, et al. [1].

\section{Discussion}

This clinical case described a very rare, atypical clinical course of CMV maternal-to-fetus infection. This presentation, limited to infra- 

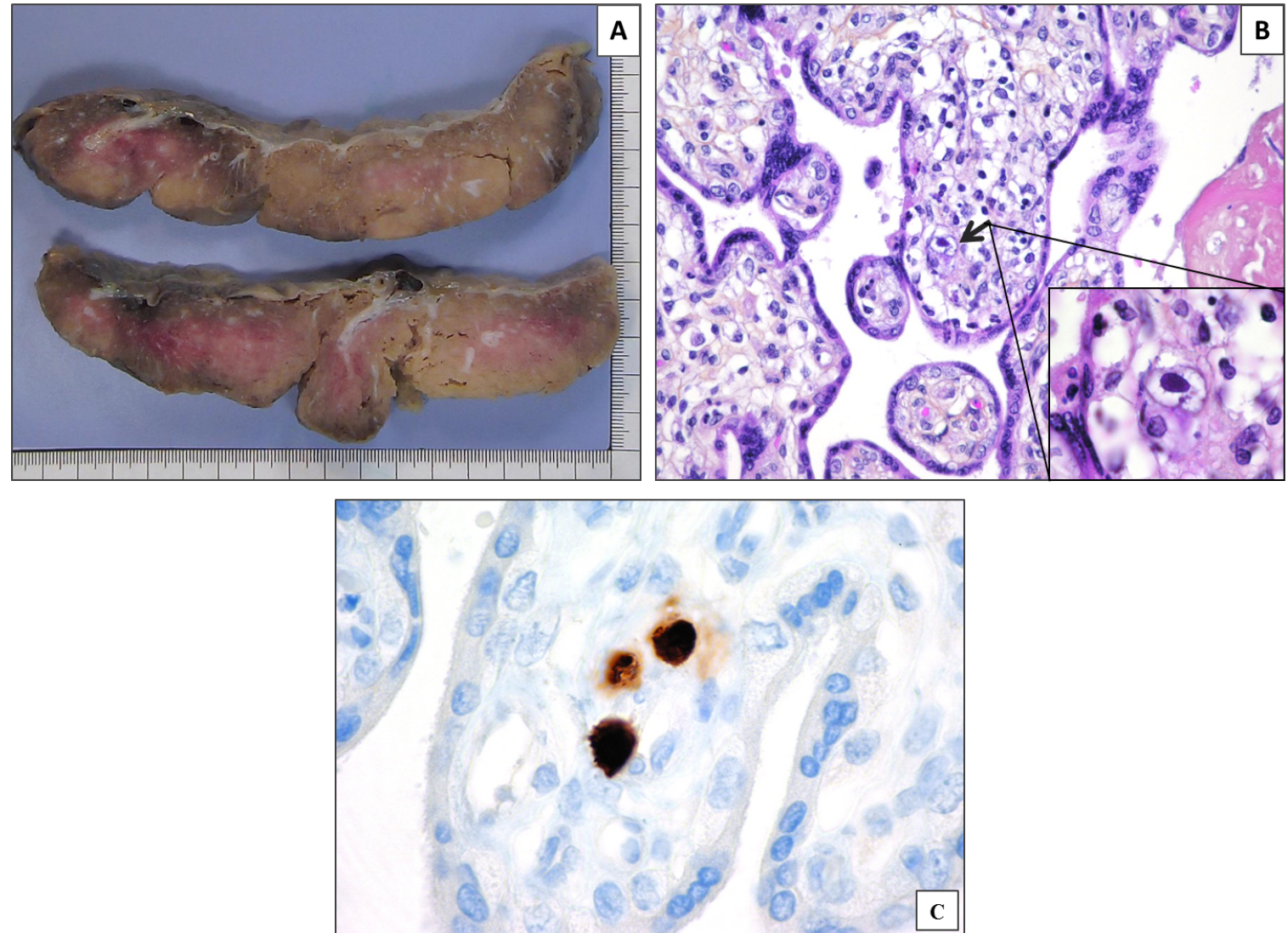

Figure 2. Pathological examination of the placenta. A) Macroscopic aspect of the placenta. The placenta was hypertrophic (600g) with an apparent pale section. Hydropics did not present any individually identifiable lesion. B) Microscopic aspect of the placenta after HPS coloration (X400 and X1000). Pathologists' examination highlighted important inflammation of villi with presence of lymphocytes and histiocytes. Cells presented large eosinophil intra-nuclear inclusion (shown by the arrow). Examination at X1000 magnification concluded to an owl-eye aspect. These arguments suggested an important viral infection through the placenta. C) Microscopic aspect of the placenta after anti-CMV antibody staining (Ventana, Argene, France) (X400). Coloration of the nuclei demonstrated the CMV nature of the inclusions.

diaphragmatic lesions, is, to our knowledge, one of the first described in the literature. More than just the case report, this clinical course highlighted some important questions about CMV management in pregnant woman.

CMV is one of the most important pregnancy-impacting viruses with an estimated prevalence of less than $1 \%$ of the newborns [2]. Search for CMV is fundamental when fetus present some alteration in cephalic pole development, to limit neurological development abnormalities. This abnormality exist when infection occurred during the first trimester and near $30 \%$ of the infants will suffer from neurological sequelae [3]. In this case, clinicians were misled because of the atypical and strictly intra-abdominal lesions, excluding CMV infection cause in their algorithm of decision. A significant lap in diagnosis was then observed (more than 2 months, $22^{\text {th }}$ to $31^{\text {th }}$ of amenorrhea) between the first observation of the cystic lesion and the diagnosis of CMV nature of the infection. Moreover, an important impact on the parents' life, associated with the need to be near a specialized maternity hospital, has been deplored. Before infectious diagnosis, it was decided to surgically cure these lesions, with important human and logistic organization. We here insisted on the fact that CMV infections not only express themselves by neurological of cephalic lesions but also by urinary and digestive alterations and sometimes only by these alterations. We have experienced, this year, in our laboratory, 2 cases confirmed of CMV infection with impact on both cephalic and abdominal pole. These two cases led to fetal deaths, despite all therapeutic used by obstetricians after diagnosis confirmation. Digestive damages, as for all non-neurological damages due to CMV, are reflect of an important dissemination of CMV inside the fetus and could be interpreted as a prognosis factor of fatal conclusion for the pregnancy [4].

Historically, research of $\mathrm{CMV}$ infection was performed using anti-CMV serology [5,6]. However, the case described in this article showed that this mode of research is not enough to strictly exclude a CMV infection and reassure the pregnant woman about the risk of contamination of her fetus. Indeed, despite of her immunity to CMV previously to her pregnancy, this patient has declared a severe and lethal materno-fetal infection with high loads of CMV in amniotic fluids and placenta. Here, we can suggest to add, systematically, search for viral presence (of herpesviridae, paramyxoviridae, and parvoviridae at least) by PCR or other molecular techniques, when a call point is found during clinical, biological or radiographic examination. At this purpose, it would be very interesting to develop and spread the use of multiplex panel in infection exploration during pregnancy. Easy-touse, quick and less expensive than at the beginning of the technique development, these diagnostic tests would be of great interest in order to optimize pregnancy management. Recently, Desveaux. et al. have suggested the utilization of a 34-peptide classifier to symptomatic to non-symptomatic newborns after CMV intra-uterine infection. This biomarker could be used after CMV infection confirmation to optimize clinical management of congenital CMV [7].

Finally, sequencing of these strains would have been very useful. Use of deep sequencing techniques has to be applied more frequently 
to these clinical situations but genomic analyses, especially in a context of fetal death were very difficult to obtain, as it was the case here. It is essential to set up clinician and scientist collaboration, to link biological and clinical metadata. These kinds of collaboration would permit to understand why some strains are associated to severe form of fetal disease and why, for example, some vertical transmission of CMV lead to neurological-only form or digestive associated form, when symptomatic. Understanding these viral characteristics, associated with more comprehension with human immune system would permit to develop therapeutic and/or vaccine and limit these type of infection.

\section{Ethics statement}

For the purpose of this study, patient confidentiality was strictly protected. Due to the retrospective nature of this study and because no supplementary sampling or analysis has been realized, no specific consent has to be obtained for the purpose of this study.

\section{Conflicts of interests}

The authors declare no conflicts of interest related to this study.

\section{References}

1. Cagdas A, Aydinli K, Irez T, Temizyürek K, Apak MY (2009) Evaluation of the fetal kidney maturation by assessment of amniotic fluid alpha-1 microglobulin levels. Eur J Obstet Gynecol Reprod Biol 90: 55-61. [Crossref]

2. Kenneson A, Cannon MJ (2007) Review and meta-analysis of the epidemiology of congenital cytomegalovirus (CMV) infection. Rev Med Virol 17: 253-276. [Crossref]

3. Pass RF, Fowler KB, Boppana SB, Britt WJ, Stagno S (2006) Congenital cytomegalovirus infection following first trimester maternal infection: symptoms at birth and outcome. J Clin Virol 35: 216-220. [Crossref]

4. Benoist G, Salomon LJ, Jacquemard F, Daffos F, Ville Y (2008) The prognostic value of ultrasound abnormalities and biological parameters in blood of fetuses infected with cytomegalovirus. BJOG 115: 823-829. [Crossref]

5. ANAES. Évaluation de l'intérêt du dépistage de l'infection à cytomégalovirus chez la femme enceinte en France. 2004 [accessed 2017 Apr 7].

6. Yinon Y, Farine D, Yudin MH1 (2018) No 240-Infection à cytomégalovirus pendant la grossesse. J Obstet Gynaecol Can 40: e142-142e150. [Crossref]

7. Desveaux C, Klein J, Leruez-Ville M, Ramirez-Torres A, Lacroix C, et al. (2016) Identification of Symptomatic Fetuses Infected with Cytomegalovirus Using Amniotic Fluid Peptide Biomarkers. PLoS Pathog 12: e1005395. [Crossref]

Copyright: (C2018 Pichon M. This is an open-access article distributed under the terms of the Creative Commons Attribution License, which permits unrestricted use, distribution, and reproduction in any medium, provided the original author and source are credited. 